\title{
Optimization of cardiac output by incremental fluid administration is associated with iatrogenic haemodilution and a paradoxical decrease in oxygen delivery
}

Liana Valeanu ${ }^{1,4}$, Serban I. Bubenek ${ }^{1,4}$, Mihai Popescu²,4, Mihai Cacoveanu ${ }^{1}$, Dana Tomescu ${ }^{2,3}$, Azriel Perel $^{4}$

1 Emergency Institute for Cardiovascular Diseases Prof. Dr. C. C. Iliescu, Anaesthesia and Intensive Care Department I, Bucharest, Romania

2 Fundeni Clinical Institute, Anaesthesia and Intensive Care Department III, Bucharest, Romania

3 Carol Davila University of Medicine and Pharmacy, Bucharest, Romania

4 Anaesthesia and Intensive Care Department, Sheba Medical Center, Tel Aviv University, Israel

Background and Goal of Study: Fluid administration may cause iatrogenic haemodilution that may eventually lead to avoidable blood transfusions [1]. We examined the effects of incremental fluid loading aimed at increasing the cardiac output (CO) on noninvasive $(\mathrm{SpHb})$ and laboratory determined $(\mathrm{BHb})$ haemoglobin levels.

Materials and Methods: After informed consent, 40 adult patients undergoing major gastrointestinal or vascular surgery were included. Oxygen saturation $\left(\mathrm{SpO}_{2}\right)$ and $\mathrm{SpHb}$ were continuously measured by a Radical-7 Pulse CO-Oximeter (Masimo Inc.). BHb and $\mathrm{PaO}_{2}$ were intermittently measured (ABL800 Radiometer). $\mathrm{CO}$ and stroke volume (SV) were continuously measured using a minimally invasive uncalibrated $\mathrm{CO}$ monitoring system (Vigileo, Edwards). Oxygen delivery $\left(\mathrm{DO}_{2}\right)$ was calculated as $\mathbf{C O} \mathbf{x}$ $\left(\left(\mathbf{B H b x 1 . 3 8 x S p O}_{2}\right)+\left(\mathrm{PaO}_{2} \mathbf{x 0 . 0 0 3 1}\right)\right)$. Baseline values were recorded after induction of anaesthesia (T0) and $5 \mathrm{~min}$ after the administration of a $250 \mathrm{ml}$ colloid fluid challenge (FC) (T1). In patients whose SV increased $\geq 10 \%$ compared to $\mathrm{T} 0$, another FC was given and all parameters recorded 5 minutes after its completion (T2). The same protocol was repeated at T2 and T3 values were obtained. Student's paired and unpaired tests, Wilcoxon signed rank test and ANOVA were used where appropriate.

Results and Discussion: All 40 patients received one FC (T1), 32 patients received 2 FCs (T2), and 20 patients received 3 FCs (T3). Figure 1 represents the median values of $\mathrm{SpHb}, \mathrm{BHb}, \mathrm{CO}$ and $\mathrm{DO}_{2}$ and their percentiles during the study and the number of patients studied at each time point. There was a statistically significant decrease in $\mathrm{SpHb}$ and $\mathrm{BHb}$ after each $\mathrm{FC}$ associated with a significant increase of $\mathrm{CO}$ and $\mathrm{DO}_{2}$ in comparison with $\mathrm{T} 0$ values.
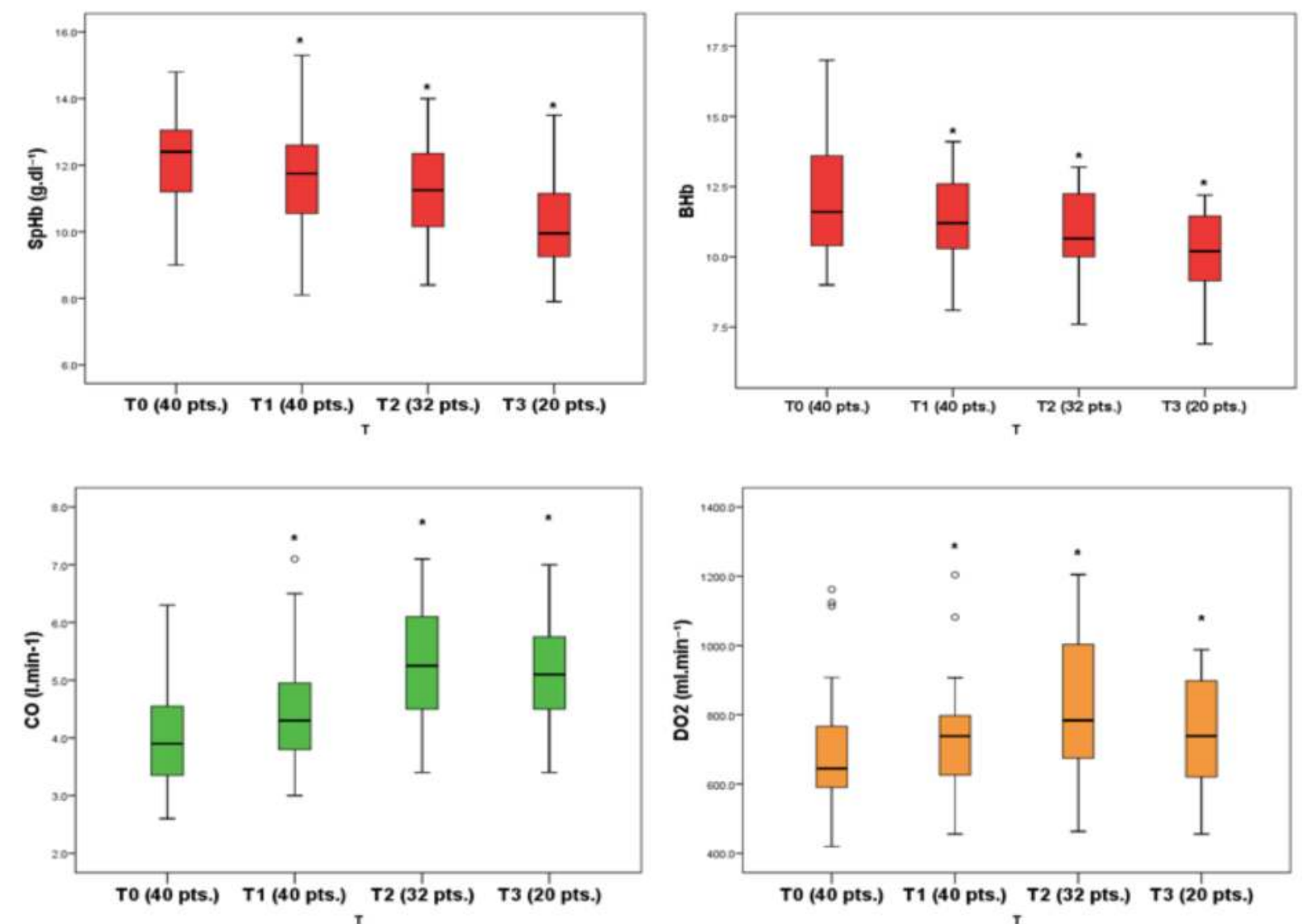

Figure 1: $\mathrm{SpHb}, \mathrm{BHb}, \mathrm{CO}$ and $\mathrm{DO}_{2}$ values during the study.

Among the 20 patients who received $3 \mathrm{FCs}$ and reached T3, the $\mathrm{CO}$ and the DO2 increased between T0-T1 and T1-T2. However, after the last $\mathrm{FC}$ the $\mathrm{CO}$ remained unchanged but the $\mathrm{DO} 2$ decreased significantly (T2-T3, figure 2). The $\mathrm{SpHb}$ and $\mathrm{BHb}$ decreased similarly after each FC (Figures 2 and 3). The decrease in $\mathrm{SpHb}$ and the $\mathrm{BHb}$ after the infusion of $750 \mathrm{ml}$ colloids was $1.66 \pm 0.67$ and $1.7 \pm 0.7 \mathrm{~g} / \mathrm{dL}$, respectively.
This significant decrease in $\mathrm{Hb}$ values explains the observed decrease in the DO2. There was a gradual increase in the mean SV between T0-T1, T1-T2, and T2-T3. However, the DO2 remained unchanged between $\mathrm{T} 0-\mathrm{T} 1$, increased significantly only from $\mathrm{T} 1-\mathrm{T} 2$ and decreased significantly between $\mathrm{T} 2$ and $\mathrm{T} 3$.
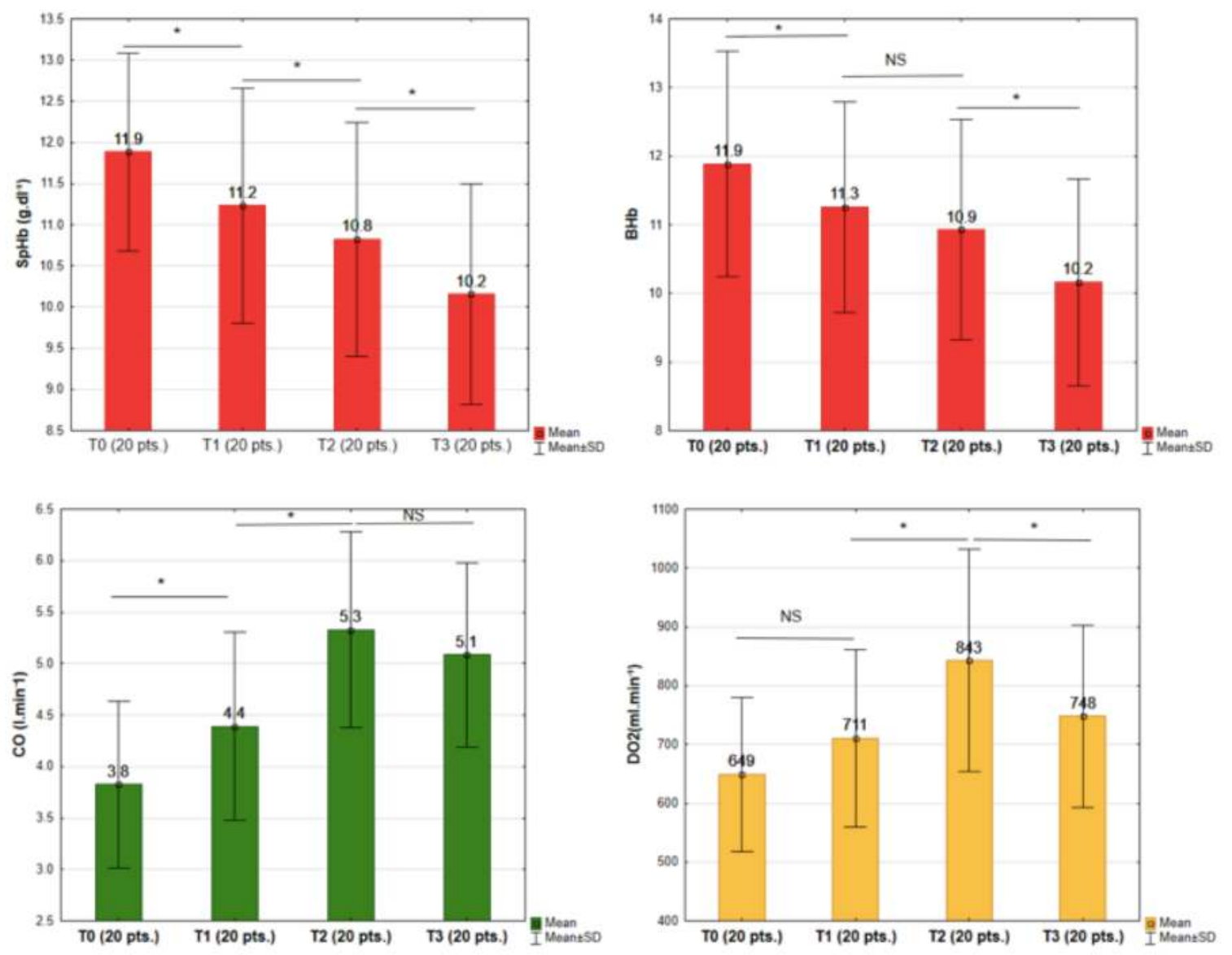

Figure 2: Evolution of $\mathrm{SpHb}, \mathrm{BHb}, \mathrm{CO}$ and $\mathrm{DO}_{2}$ values in patients receiving 3 fluid challenges (T3)

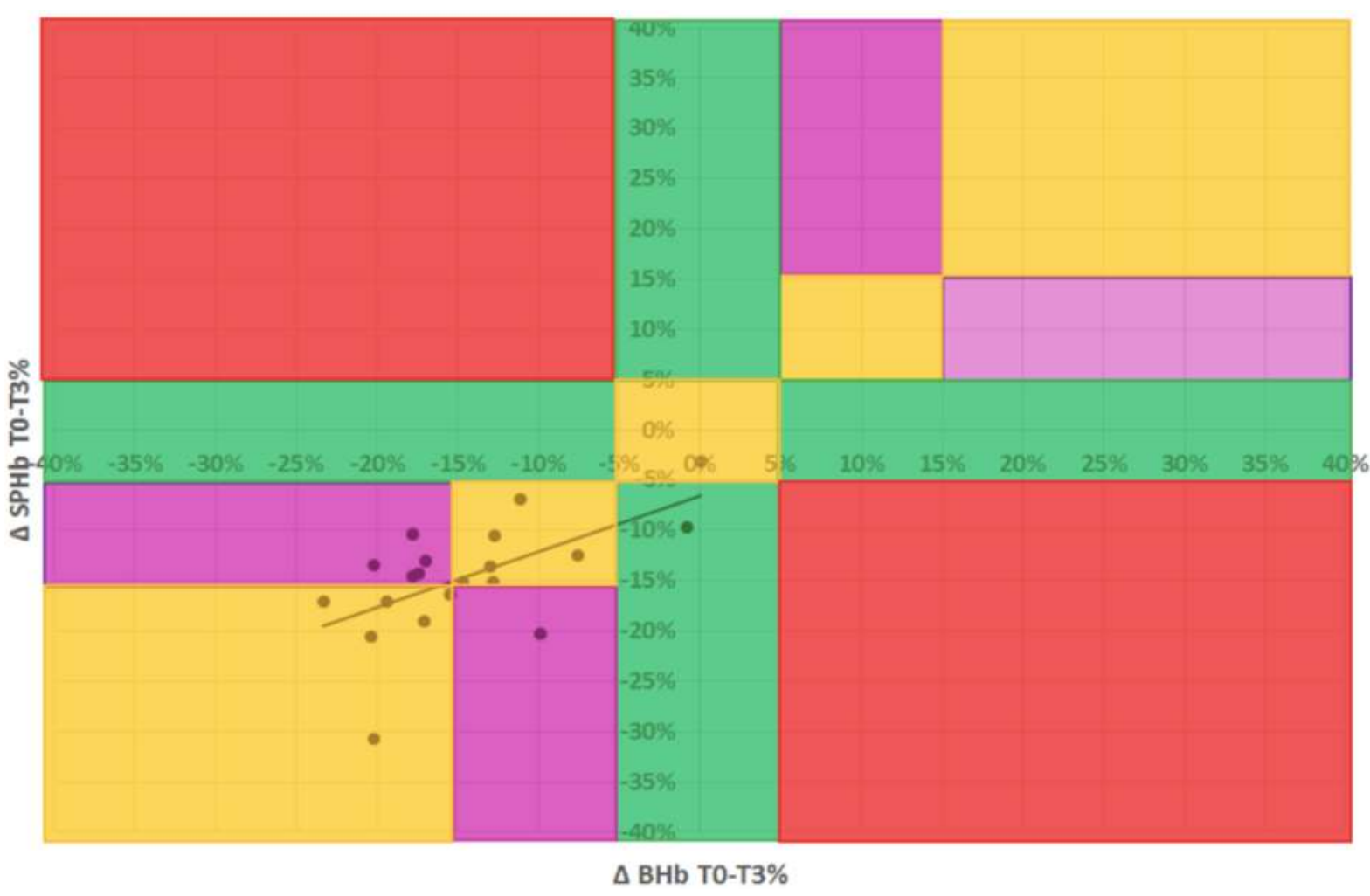

Figure 3: Error grid plot based on clinical concordance categories defining the level of agreement between $\triangle \mathrm{SpHb}$ and $\triangle \mathrm{BHb}$ data pairs in patients who received $3 \mathrm{FC}$ (T3). Zone 1 (yellow): $\mathrm{SpHb}$ and $\mathrm{BHb}$ change in the same direction and to the same extent, resulting in correct treatment decisions. Zone 2 (magenta): $\mathrm{SpHb}$ and $\mathrm{BHb}$ change in the same direction but not to the same extent, resulting in exaggerated or insufficient therapy. Zone 3 (green): SpHb changes while $\mathrm{BHb}$ is constant or vice versa, reflecting unnecessary or necessary but withheld therapy . Zone 4 (red): opposite changes in $\mathrm{SpHb}$ and $\mathrm{BHb}$, resulting in opposite treatment.

Conclusions: Fluid loading in goal-directed therapy may cause a paradoxical decrease in $\mathrm{DO}_{2}$ due to iatrogenic haemodilution. The continuous monitoring of $\mathrm{SpHb}$ provides real-time reflection of hemodilution. The changes observed in the $\mathrm{SpHb}$ trend are similar to the changes in intermittent $\mathrm{BHb}$ values. 